\title{
Agressão sexual entre jovens universitários: questão de saúde pública?
}

| ${ }^{1}$ Lylla Winzer I

${ }^{1}$ Institute for Population and Social Research. Mahidol University, Thailand (lylla.winzer@yahoo.com).

Os recentes casos de estupro na Universidade de São Paulo (CAMBRICOLI, 2014; SOARES, 2014) fomentaram a discussão, no Brasil, sobre o tema da agressão sexual em estudantes universitários. Apesar de o assunto ter sido até então negligenciado no país, o problema não parece ser novidade para pesquisadores da área.

Uma revisão bibliográfica em cinco diferentes bases de dados (Scielo, PubMed, Scopus, PsycINFO e Web of Knowledge) revela que pelo menos cinco estudos têm abordado a ocorrência de agressão sexual exclusivamente em estudantes universitários no país (CHAN et al., 2008; D'ABREU; KRAHÉ; BAZON, 2013; FLAKE et al., 2013; HINES, 2007; ZOTARELI et al., 2012). Focando em alunos no primeiro ano de faculdade, um estudo mostrou que $29 \%$ das moças e $27 \%$ dos rapazes já haviam sido vítimas de alguma forma de agressão sexual desde os 14 anos (a idade de consentimento no Brasil). Entre as vítimas, a grande maioria dos homens (83\%) e mulheres (93\%) revelou que a agressão foi perpetrada por alguém conhecido, incluindo parceiro fixo ou casual. Se discriminados os casos que atenderiam à definição legal de estupro segundo o Código Penal Brasileiro, as taxas seriam de $8 \%$ para as mulheres e de $7,4 \%$ para os homens (D'ABREU; KRAHÉ; BAZON, 2013).

Outros estudos, usando instrumento semelhante de coleta de dados, investigaram casos de agressão sexual perpetrada especificamente por parceiro íntimo nos 12 meses anteriores à pesquisa. O primeiro revelou que 38,5\% das alunas e $34,5 \%$ dos alunos já haviam sofrido alguma forma de vitimização sexual (CHAN et al., 2008). O segundo estudo mostrou que 39,5\% de estudantes do sexo feminino e $29,7 \%$ do sexo masculino revelaram terem sido vítimas de 
coerção sexual verbal no ano anterior, e que 1,3\% das mulheres e 1,4\% dos homens tinham sido vítimas de coerção sexual física (HINES, 2007). O terceiro estudo revelou que cerca de 40\% das mulheres e 37\% dos homens já havia experienciado alguma forma de vitimização sexual (FLAKE et al., 2013). E finalmente, um último estudo, que incluiu apenas estudantes do sexo feminino, encontrou uma taxa de vitimização de 9,4\% desde o início da vida universitária, cometida por agressores conhecidos ou não (ZOTARELI et al., 2012); uma porcentagem ainda alta, mesmo que inferior às taxas de estudos anteriores.

Quatro dos estudos anteriormente mencionados também investigaram o autorrelato de perpetração de agressão sexual. As taxas de homens que assumiram alguma forma de perpetração de agressão sexual, incluindo estupro, variaram de 3,3\% até 43,7\% (CHAN et al., 2008; D'ABREU; KRAHE; BAZON, 2013; FLAKE et al., 2013; ZOTARELI et al., 2012). Entre as mulheres, essas taxas variaram de 3\% a 31,6\% (CHAN et al., 2008; D'ABREU; KRAHÉ; BAZON, 2013; FLAKE et al., 2013).

Apesar de a maior parte dos estudos mostrar que parceiro íntimo é perpetrador comum, eles não fornecem dados qualitativos sobre as diferenças entre agressão perpetrada por (ex-) parceiros ou por desconhecidos. Um estudo sugere ainda que orientação sexual pode estar relacionada à maior vulnerabilidade. Homens e mulheres que se definiram como homossexuais ou bissexuais apresentaram taxas mais altas de vitimização (D'ABREU; KRAHÉ; BAZON, 2013). No entanto, por se tratar de uma amostra de tamanho pequeno, os resultados não são conclusivos.

Outro dado saliente é que vítimas e perpetradores incluem tanto mulheres quanto homens. Com os papéis de gênero cada vez mais semelhantes entre jovens (PAUL; HAYES, 2002), mulheres têm tomado com maior frequência a iniciativa na relação sexual, o que pode em parte explicar um número alto de homens reportando diferentes formas de vitimização sexual (D'ABREU; KRAHE; BAZON, 2013). Esses dados desafiam o mito de que homens não podem ser vitimizados sexualmente e que mulheres não podem ser perpetradoras.

Uma pesquisa mostrou que, entre homens vitimizados que reportaram o sexo de seus perpetradores, a grande maioria (72\%) indicou apenas mulheres como autoras da agressão. Outros $12 \%$ reportaram que foram vitimizados por agressores de ambos os sexos e 16\% indicaram que seus perpetradores foram 
somente homens (D'ABREU; KRAHE; BAZON, 2013). É verdade, contudo, que a agressão feminina ao homem não relativiza a violência masculina contra mulheres. Esta parece ter impacto mais severo, já que há indícios de que o impacto da agressão sexual em homens é percebido apenas como moderado (KRAHÉ; SCHEINBERGER-OLWIG; BIENECK, 2003).

Apesar da variação da incidência do problema devido à diferença nos instrumentos usados, idade da amostra e local onde a pesquisa foi realizada, os resultados parecem confirmar que estudantes universitários estão em risco para agressão sexual. A vida universitária é caracterizada por maior autonomia e maior experimentação sexual (CAETANO et al., 2010), o que pode aumentar significativamente a probabilidade de ocorrência de agressão sexual (MALAMUTH et al., 1991). Especial atenção deve ser dedicada ao consumo excessivo de álcool e drogas e à banalização da pornografia entre jovens.

Um estudo brasileiro mostrou que o consumo de álcool por estudantes universitários é maior do que na população em geral (ANDRADE; DUARTE; OLIVEIRA, 2010), e há evidência de que a maioria dos casos de agressão sexual envolve consumo de álcool pela vítima e/ou pelo perpetrador (ABBEY et al., 2004). Outro fator importante, mas pouco estudado no Brasil, referese ao consumo de pornografia. Estudos transversais e longitudinais brasileiros mostram que o consumo de pornografia age com importante preditor tanto para agressão quanto vitimização sexual (D'ABREU, 2013; D’ABREU; KRAHÉ, 2014; D'ABREU; KRAHÉ, 2015). O complexo mecanismo que explica como consumo de álcool e de pornografia está relacionado à agressão sexual pode ser acessado em outras publicaçôes (ABBEY et al., 2004; D'ABREU, 2013; D'ABREU; KRAHÉ, 2014; D'ABREU; KRAHÉ, 2015).

Há muito tempo a agressão sexual em jovens universitários tem sido negligenciada, provavelmente por se tratar de um grupo social privilegiado. As pesquisas sobre violência sexual no Brasil são em grande parte baseadas em amostras clínicas do SUS ou em amostras da comunidade com maior vulnerabilidade socioeconômica. Violência sexual entre jovens de classe média e alta parece ser percebida como um problema menor, especialmente se o perpetrador é (ex-) parceiro íntimo ou se houve qualquer sinal de má conduta pela vítima, como abuso de álcool ou drogas e promiscuidade (WARSHAW; KOSS, 1988). 
No entanto, pelas altas taxas de incidência e reincidência e pelo impacto na saúde reprodutiva dos estudantes (complicações ginecológicas, proliferação de DSTs e impacto na saúde mental), a questão tem sido apontada com um importante problema de saúde pública em populações de estudantes universitários (DEGUE, 2014). Ainda não há estudos disponíveis no Brasil sobre o problema em outros estados, além de São Paulo. Mas como os fatores de risco são comuns a universitários de forma geral, há motivos para se crer que o problema atinja milhares de jovens em todo o país.

Ao atribuir a responsabilidade da questão apenas às instituiçôes de ensino, o Ministério Público exime o governo do seu dever. É importante que governos e universidades ajam juntos na prevenção do problema entre os estudantes, na atenção aos grupos que apresentam maior risco e na atenção a vítimas e perpetradores. A experiência norte-americana aponta que o problema é passível de prevenção (DEGUE, 2014) e que existem programas bem-sucedidos que podem ser replicados no Brasil.

Conduzir pesquisas, implementar programas educacionais, promover comportamentos pró-sociais e recomendar políticas públicas são maneiras de prevenir a ocorrência ou recorrência do problema. Sob o ponto de vista da saúde pública, é imprescindível que governos, universidades, pesquisadores e a sociedade reconheçam, pela magnitude do problema, que se trata de uma questão grave, e que sejam capazes de identificar e intervir em variáveis preditoras e nas potenciais consequências físicas e psicossociais, tanto para vítimas como para perpetradores.

\section{Referências}

ABBEY, A. et al. Sexual assault and alcohol consumption: What do we know about their relationship and what types of research are still needed? Aggression and Violent Behavior, v. 9, n. 3, p. 271-303, 2004.

ANDRADE, A. G.; DUARTE, P.; OLIVEIRA, L. G. D. I levantamento nacional sobre o uso de álcool, tabaco e outras drogas entre universitários das 27 capitais brasileiras. Brasília: Secretaria Nacional de Políticas sobre Drogas, 2010. Disponível em:<http://nute.ufsc.br/bibliotecas/ upload/ilevantamentodrogasuniversitarios_-_pt-br_2010.pdf> Acesso em: 26 jun. 2015.

CAETANO, M. E. et al. Sexual behavior and knowledge of sexually transmitted infections among university students in São Paulo, Brazil. International Journal of Gynaecology \& Obstetrics, v. 110, n. 1, p. 43-6, 2010. 
CAMBRICOLI, F. Faculdade de Medicina da USP tem dois novos casos de estupro. O Estado de São Paulo, São Paulo, 25 nov. 2014. Disponível em: <http://sao-paulo.estadao.com.br/ noticias/geral,faculdade-de-medicina-da-usp-tem-dois-novos-casos-de-estupro,1598003> Acesso em: 26 jun. 2015.

CHAN, K. L. et al. Prevalence of dating partner violence and suicidal ideation among male and female university students worldwide. Journal of Midwifery \& Women's Health, v. 53, n.6, p. 529-37, 2008.

D'ABREU, L. C. F. Pornografia, desigualdade de gênero e agressão sexual contra mulheres. Psicologia e Sociedade, v. 25, n. 3, p. 592-601, 2013.

D'ABREU, L. C. F.; KRAHÉ B. Vulnerability to sexual victimization in female and male college students in Brazil: Cross-sectional and prospective evidence. Archives of Sexual Behavior, p. 1-15, 2015.

D'ABREU, L. C. F.; KRAHÉ B. Predicting sexual aggression in male college students in Brazil. Psychology of Men and Masculinity, v. 15, n. 2, p. 152, 2014.

D'ABREU, L. C. F.; KRAHÉ, B.; BAZON, M. R. Sexual aggression among Brazilian college students: prevalence of victimization and perpetration in men and women. Journal of Sex Research, v. 50, n. 8, p. 795-807, 2013.

DEGUE, S. Preventing sexual violence on college campuses: lessons from research and practice. Disponível em:<https://www.notalone.gov/assets/evidence-based-strategies-forthe-prevention-of-sv-perpetration.pdf> Acesso em: 26 jun. 2015.

FLAKE, T. A. et al. Intimate partner violence among undergraduate students of two universities of the state of São Paulo, Brazil. Revista Brasileria de Epidemiologia, v. 16, n. 4, p. 801-16, 2013.

HINES, D. A. Predictors of sexual coercion against women and men: a multilevel, multinational study of university students. Archives of Sexual Behavior, v. 36, n. 3, p. 403-22, 2007.

KRAHÉ, B.; SCHEINBERGER-OLWIG, R.; BIENECK, S. Men's reports of nonconsensual sexual interactions with women: prevalence and impact. Archives of Sexual Behavior, v. 32, n. 2, p. 165-75, 2003.

MALAMUTH, N. M. et al. Characteristics of aggressors against women: testing a model using a national sample of college students. Journal of Consulting and Clinical Psychology, v. 59, n. 5, p. 670, 1991.

PAUL, E. L.; HAYES, K. A. the casualties of 'casual' sex: a qualitative exploration of the phenomenology of college students' hookups. Journal of Social and Personal Relationships, v. 19, n. 5, p.639-61, 2002. 
SOARES, L. Alunas denunciam estupro em festas da Faculdade de Medicina da USP. Folha de São Paulo, São Paulo, 13 jun. 2014. Disponível em: <http://www1.folha.uol.com. $\mathrm{br} /$ cotidiano/2014/11/1546868-alunas-denunciam-estupros-em-festas-da-faculdade-demedicina-da-usp.shtml> Acesso em: 26 jun. 2015.

WARSHAW, R.; KOSS, M. I never called it rape. New York: Harper \& Row Publishers, 1988. 256p.

ZOTARELI, V. et al. Gender and sexual violence among students at a Brazilian university. Revista Brasileira de Saúde Materno-Infantil, v. 12, p. 7-46, 2012. 